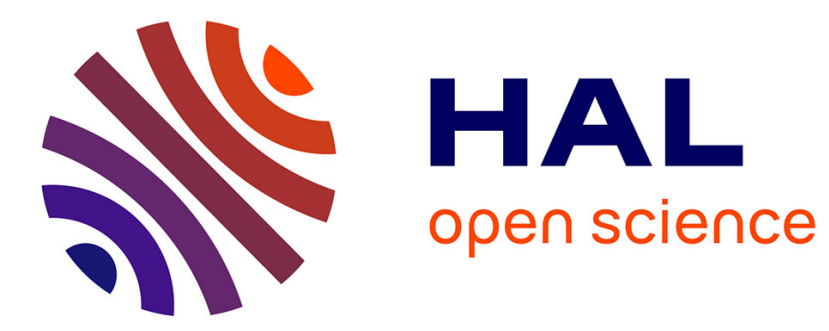

\title{
Steps Towards Safe Navigation in Open and Dynamic Environments
}

\author{
Stephane Petti, Dizan Alejandro Vasquez Govea, Manuel Yguel, Thierry \\ Fraichard, Olivier Aycard
}

\section{- To cite this version:}

Stephane Petti, Dizan Alejandro Vasquez Govea, Manuel Yguel, Thierry Fraichard, Olivier Aycard. Steps Towards Safe Navigation in Open and Dynamic Environments. Laugier, Christian and Chatila, Raja. Autonomous Navigation in Dynamic Environments, 35, Springer, 2007, Springer Tracts in Advanced Robotics. inria-00182029v3

\section{HAL Id: inria-00182029 https://hal.inria.fr/inria-00182029v3}

Submitted on 20 Mar 2008

HAL is a multi-disciplinary open access archive for the deposit and dissemination of scientific research documents, whether they are published or not. The documents may come from teaching and research institutions in France or abroad, or from public or private research centers.
L'archive ouverte pluridisciplinaire HAL, est destinée au dépôt et à la diffusion de documents scientifiques de niveau recherche, publiés ou non, émanant des établissements d'enseignement et de recherche français ou étrangers, des laboratoires publics ou privés. 


\title{
Steps Towards Safe Navigation in Open and Dynamic Environments
}

\author{
C. Laugier, S. Petti, D. Vasquez, M. Yguel, Th. Fraichard \& O. Aycard \\ INRIA Rhône-Alpes \& GRAVIR Lab. (CNRS, INPG, UJF) \\ http://emotion.inrialpes.fr
}

\begin{abstract}
Autonomous navigation in open and dynamic environments is an important challenge, requiring to solve several difficult research problems located on the cutting edge of the state of the art. Bassically, these problems can be classified into three main categories: SLAM in dynamic environments; Detection, characterization, and behavior prediction of the potential moving obstacles; On-line motion planning and safe navigation decision based on world state predictions. This paper addresses some aspects of these problems and presents our latest approaches and results. The solutions we have implemented are mainly based on the followings paradigms: Characterization and motion prediction of the observed moving entities using bayesian programming; $O n$ line goal-oriented navigation decisions using the Partial Motion Planning $(P M P)$ paradigm.
\end{abstract}

\section{INTRODUCTION}

\section{A. Outline of the problem}

To some extent, autonomous navigation for robotic systems placed in stationary environments is no longer a problem. The challenge now is autonomous navigation in open and dynamic environments, i.e. environments containing moving objects (potential obstacles) whose future behaviour is unknown. Taking into account these characteristics requires to solve several difficult research problems at the cutting edge of the state of the art. Basically, these problems can be classified into three main categories:

- Simultaneous Localisation and Mapping (SLAM) in dynamic environments;

- Detection, tracking, identification and future behaviour prediction of the moving obstacles;

- On-line motion planning and safe navigation

In such a framework, the system has to continuously characterize the fixed and moving objects that can be observed both with on-board or off-board sensors. As far as the moving objects are concerned, the system has to deal with problems such as interpreting appearances, disappearances, and temporary occlusions of rapidly manoeuvring objects. It also has to reason about their future behaviour (and consequently to make predictions). From the autonomous navigation point of view, this means that the system has to face a double constraint: constraint on the response time available to compute a safe motion (which is clearly a function of the dynamicity of the environment), and a constraint on the temporal validity of the motion planned (which is a function of the validity duration of the predictions). In other words, one needs to be able to plan motion fast, but one does not need to plan motion very far in the future.
This paper addresses some aspects of the previous problem, and presents our latest approaches and results. The solutions we have implemented rely on the following modules:

- Scene interpretation and short-term motion prediction for the moving obstacles, using the new concept of Bayesian Occupancy Filters and an efficient waveletbased representation of the related occupancy grids;

- Medium-term motion and behaviour prediction for the observed moving objects, using motion pattern learning and Hidden Markov Models;

- On-line goal-oriented navigation decision using the Partial Motion Planning (PMP) paradigm.

\section{B. Case Study: The Automated Valet Parking}

One possible (and very relevant) target application for the techniques presented in this paper is that of the Automated Valet Parking (AVP). The robotic system considered is a "smart" car operating autonomously in a "smart" city parking. Both the car and the parking are equipped with sensors providing them with information about the world. Let us imagine the following scenario: you drive your car and leave it at the entrance of a given parking. From then on, it operates autonomously and go park itself. As soon as the car enters the parking, the car on-board intelligent system connects to the parking's own intelligent system and request a free parking place. The parking then confirms the availability of a parking space and provides the car with a model of the parking and an itinerary to the empty place. From then on, the car, using information obtained from both its own sensors and the parking sensory equipment, go park itself.

From an architecture point of view, the AVP scenario involves two "intelligent" systems communicating with one another: the car on-board system and the parking off-board system. As mentioned earlier, it is assumed that both systems are equipped with sensors providing them with information about the environment considered. While the car sensors will provide it with a local view of its surroundings, it can be expected that the parking sensors will provide the car with an overall view of what is going on in the whole parking.

To address the AVP scenario, we have devised a scheme relying upon the following functionalities (split between the car and the parking)

Parking abilities:

- Parking monitoring: at any time, the parking should know which places are free or not (and by whose car they are occupied). 
- Route planning: the parking should be able to provide the car with a model of the parking premises along with the best itinerary to reach a given place.

- Moving objects monitoring: any moving objects (vehicles, pedestrians, etc.) should be monitored and tracked. The parking should be able to provide information such as position, speed and expected trajectory (i.e. future behaviour). Expected trajectories can come from different clues: typical movements of the different kinds of moving objects, learnt from previous observation, or knowledge of a planned trajectory.

- Car localisation: given its moving objects' monitoring functionality, the parking can provide the car with its current state in the parking premises.

Car abilities:

- Localisation: the car should be able to maintain an estimate of its localisation in the parking. It can be the result of a data fusion between parking information and on-board localisation.

- Environment modelling: the car on-board sensor are primarily used to build a model of the surroundings of the car. This local model should be enriched using the global information provided by the parking (in particular, the information concerning the moving objects' future behaviour).

- Automated driving: given the parking model and the route to the goal, the car should be able to determine its future course of action so as to reach its goal efficiently and safely.

One can notice that some of the functionalities mentioned above are somewhat redundant (in particular when dealing with sensing data). This property has intentionally been chosen in order to increase the robustness and the efficiency of the system:

- Fusion of data from multiple source increase overall accuracy.

- Using several data source increase fault tolerance.

- By correlating different inputs, it is possible to diagnose if an input is failing or becoming unreliable.

\section{Outline of the paper}

In this paper, we focus on two of the functionalities mentioned in the previous section: Motion prediction of the observed moving objects and on-line goal-oriented navigation decisions. The paper is organized in three main sections. The section II describes how we have solved the problem of interpreting and representing the dynamic environment of the robot using the "Bayesian Occupancy Filtering" $(B O F)$ approach; this approach relies on a local world-state bayesian interpretation scheme, including a short-term motion prediction mechanism. The section III deals with the problem of the prediction of the most likely behaviors of some observed objects executing "intentional motions"; the proposed solution relies on the use of a motion pattern learning mechanism and of a hierarchical Hidden Markov Model. The section IV deals with the problem of planning safe motions in a reconstructed dynamic environment; the proposed paradigm (called "Partial
Motion Planning", or $P M P$ ) takes into account (at each iteration step) both the time constraints and the current model of the future state of the robot environment.

\section{SCENE INTERPRETATION AND SHORT-TERM MOTION PREDICTION}

\section{A. Overview of the problem}

The problem addressed in this section concerns the interpretation of the observed dynamic scene in terms of potential moving obstacles, i.e. obstacles which may generate a collision in the near future with the robot). The objective is to be able to correctly interpret the dynamic scene in the presence of noisy or missing data, and to be as robust as possible to temporary or partial occlusions. Our approach for solving this problem is based on the new concept of Bayesian Occupancy Filtering (BOF) [1], where the robot environment is represented using a 4-dimensional occupancy grid, i.e. an occupancy grid which includes the velocity dimension.

The occupancy grids [2], [3] framework is a classical way to describe the environment of a mobile robot. It has extensively been used for static indoor mapping [4] using a 2-dimensional grid. More recently, occupancy grids have been adapted to track multiple moving objects [5]. However, a major drawback of these approaches, is that a moving object may be lost due to occlusion effects.

The $B O F$ approach avoid this problem for short temporary occlusions (e.g. a few seconds), by combining two complementary phases in a recursive loop: the estimation phase which estimate the occupancy probability of each cell of the 4dimensional grid, using recursively the set of sensor observations; the prediction phase which estimate an a priori model of the grid occupancy at time $k+1$, using a "dynamic model" and the latest estimation of the grid state (figure 2 illustrates). This approach has been developed using the Bayesian Programming Framework [6], [7], [8]; it is described in the next sections.

However, large scale environments can hardly been processed in real-time because of the intrinsic complexity of the related inferences and numerical computations (see section IIF). The section II-G presents the outline of the $W O G$ model ("Wavelet Occupancy Grid") we are developing for trying to meet the required efficiency property.

\section{B. Estimation of the occupancy probability}

The estimation phase consists in estimating, at each time step, the occupancy probability of each cell of the 4dimensional grid. This estimation is performed using recursively the set of "observations" (i.e. pre-processed sensors data) provided by the sensors at each time step. These observations are represented by a list of detected objects, along with their associated positions and velocities in the reference frame of the processed sensor (several sensors may be used in parallel). In practice, this set of observations could also contain two types of false measurements : the false alarms, i.e. when the sensor detects a non existing object; the missed detection, i.e. when the sensor does not detect an existing object.

Solving the related estimation problem is done by using the available instantaneous information about the environment 
state (i.e. the current observations and grid state). A sketch of the algorithm is given below using our Bayesian Programming Framework [6], [7], [8]; a more complete description of the method (which includes a "sensor data fusion" step) can be found in [1].

(i) Choosing the relevant variables and decomposition.

- $O_{x, y, v_{x}, v_{y}}$ : The occupancy of the cell $\left(x, y, v_{x}, v_{y}\right)$ at time $t$ : occupied or not. This variable is indexed by a $4-$ dimensional index that represents a position and a speed relative to the vehicle.

- $\mathcal{Z}$ : The sensor observation set; one observation is denoted $Z_{s}$; the number of observation is denoted $S$.

If we make the reasonable assumption that all the observations of the sensors are independent when knowing the occupancy state of a cell, we can choose to apply the following decomposition of the related joint distribution :

$$
P\left(O_{x, y, v_{x}, v_{y}}, \mathcal{Z}\right)=P\left(O_{x, y, v_{x}, v_{y}}\right) \times \prod_{s=1}^{S} P\left(Z_{s} \mid O_{x, y, v_{x}, v_{y}}\right)
$$

(ii) Assigning the parametric forms.

According to our knowledge of the problem to be solved, we can assign the following parametric forms to each of the terms of the previous decomposition:

- $P\left(O_{x, y, v_{x}, v_{y}}\right)$ represents the a priori information on the occupancy of each cell. If available, a prior distribution could be used to specify it. Otherwise, a uniform distribution has to be selected. The next section will show how the prior distribution may be obtained from previous estimations.

- The shape of $P\left(Z_{s} \mid O_{x, y, v_{x}, v_{y}}\right)$ is given by the sensor model. Its goal is to model the sensor response knowing the cell state. Details about this model can be found in [3]; an example is given for a telemetric sensor in Fig. 1. Such models can easily been built for any kind of sensor.

(iii) Solution of the problem.

It is now possible to ask the Bayesian question corresponding to the searched solution (i.e. the searched probability distribution). Since the problem to solve consists in finding a good estimate of the cell occupancy, the question can be stated as follows :

$$
P\left(O_{x, y, v_{x}, v_{y}} \mid \mathcal{Z}\right) ?
$$

The result of the related Bayesian inference ${ }^{1}$ can be written as follows :

$$
P\left(O_{x, y, v_{x}, v_{y}} \mid \mathcal{Z}\right) \propto \prod_{s=1}^{S} P\left(Z_{s} \mid O_{x, y, v_{x}, v_{y}}\right) .
$$

\section{The Bayesian Occupancy Filter}

We are now interested in taking into account the sensor observation history, in order to be able to make more robust estimations in changing environments (i.e. in order to be able to process temporary objects occlusions and detection

\footnotetext{
${ }^{1}$ This result is computed using our ProBT inference engine, currently commercialized by our spin-off company Probayes.
}

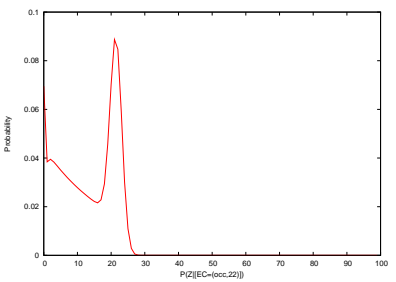

(a) Sensor model for occupied cells

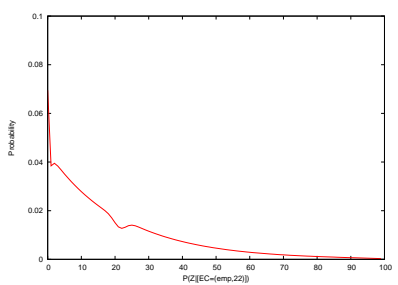

(b) Sensor model for empty cells
Fig. 1. Example of one-dimensional sensor models. The sensor is a laserrange fi nder located in $x=0$ and detecting an object at $x=22$. The following property holds : $P\left(Z \mid\left[O_{x}=o c c\right]\right)=P\left(Z \mid\left[O_{x}=e m p\right]\right)$ for $x>z$, which implies that $P\left(O_{x} \mid Z\right)$ is unknown when the cell is behind the obstacle.

problems). A classical way to solve this problem is to make use of Bayes filters [9]. Basically, the goal of such a filtering process is to recursively estimate the probability distribution $P\left(O_{x, y, v_{x}, v_{y}}^{k} \mid Z^{k}\right)$, known as the posterior distribution. In general, this estimation is done in two stages: a prediction stage whose purpose is to compute an a priori estimate of the target's state known as the prior distribution; an estimation stage whose purpose is to compute the posterior distribution, using this a priori estimate and the current measurement of the sensor. Exact solutions to this recursive propagation of the posterior density do exist in a restrictive set of cases (such as the Kalman filter [10][11] when linear functions and gaussian distributions are used).

Our approach for solving this problem, is based on the new concept of Bayesian Occupancy Filter (BOF); Fig. 2 shows the related estimation loop. This approach is derived from the general bayesian filtering paradigm; it provides a powerfull formalism for solving difficult estimation problems expressed in our 4-dimensional occupancy grid representation.

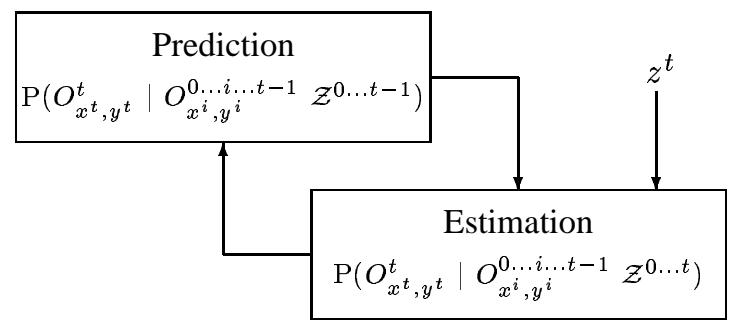

Fig. 2. Bayesian Occupancy Filter as a recursive loop.

The basic idea underlying the conception of the $B O F$ is to make use of the velocities measured in the past, for predicting the near future and propagating this information through time. Indeed, knowing the current velocity of a mobile, it is possible to predict its future motion under the hypothesis of a constant velocity for the next time step (in practice, possible velocity changes will generate several possible future positions).

A complete presentation of the $B O F$ can be found in [1]. In the sequel, we will just give an overview of the approach under the following simplifying assumptions (for clarity reasons) : use of a single sensor and constant velocity for the observed moving objects. A consequence of this last assumption, is that 
we can deal with a single "antecedent cell" when evaluating the occupancy state of a given cell.

(i) Choosing the relevant variables and decomposition.

- $O_{x, y}^{t}$ : Occupancy of the cell $c=\left(x, y, v_{x}, v_{y}\right)$ at time $t$, occupied or not.

- $O_{x_{p}, y_{p}}^{t-\delta t}$ : Occupancy of the cell which is the antecedent of $C_{x, y}^{t}$, occupied or not. In this model, $x_{p}=x-v_{x} \delta t$ and $y_{p}=y-v y \delta t$, since the velocity is constant.

- $Z_{s}$ : A sensor observation.

Under the previous simplifying assumptions, the following decomposition of the joint distribution determined by these variables can be expressed as follow:

$$
\begin{aligned}
P\left(O_{x_{p}, y_{p}}^{t-\delta t}, O_{x, y}^{t}, Z_{s}\right)= & \left(\begin{array}{l}
P\left(O_{x_{p}, y_{p}}^{t-\delta t}\right) \\
\times P\left(O_{x, y}^{t} \mid O_{x_{p}, y_{p}}^{t-\delta t}\right) P\left(Z_{s} \mid O_{x, y}^{t}\right)
\end{array}\right) .
\end{aligned}
$$

(ii) Assigning the parametric forms.

- $P\left(O_{x_{p}, y_{p}}^{t-\delta t}\right)$ is the prior for the future occupancy state of the cell $c=\left(x, y, v_{x}, v_{y}\right)$. For each cell $c$ such as the antecedent $\left(x_{p}, y_{p}, v_{x}, v_{y}\right)$ is out of the current grid, this prior is the probability that a new object enters in the monitored space; since we usually have no real information about such an event, this probability is represented by a uniform distribution.

- $P\left(O_{x, y}^{t} \mid O_{x_{p}, y_{p}}^{t-\delta t}\right)$ is related to the very simple "dynamic model" we are using in this case. It is defined as a transition matrix $\left[\begin{array}{cc}1-\epsilon & \epsilon \\ \epsilon & 1-\epsilon\end{array}\right]$, which allows the system to take in account the fact that the null acceleration hypothesis is an approximation; in this matrix, $\epsilon$ is a parameter representing the probability that the object in $c=\left(x_{p}, y_{p}, v_{x}, v_{y}\right)$ does not follow the null acceleration model.

- $P\left(Z_{s} \mid O_{x, y}^{t}\right)$ is the sensor model (see section II-B).

(iii) Solution of the problem.

Similarly to the estimation process described in the section II$\mathrm{B}$, the solution of the problem to be solved by the $B O F$ can be defined by the following Bayesian question : $P\left(O_{x, y}^{t} \mid Z_{s}\right)$ ?. Answering this question (i.e. computing the related probability distribution) is achieved using our inference engine; this inference involves a marginalization sum over $O_{x_{p}, y_{p}}^{t-\delta t}$.

\section{Experimental results}

This approach has been implemented and tested on our experimental platform : the Cycab vehicle equiped with a laser sensor. Fig. 3 shows some resulting grid estimations, for an environment containing two stationary objects and an object moving from the left to the right at a velocity of $0.8 \mathrm{~m} / \mathrm{s}$; in this example, the robot is not moving.

Fig. 3b depicts the occupancy probability of each cell corresponding to a null relative velocity (i.e. $c=[x, y, 0,0]$ ). As expected, two areas with high occupancy probabilities are visible; these probability values depends on several factors attached to the sensor model : the probability of true detections, the probability of false alarms, and the sensor accuracy.
Since the measured speed for the third obstacle is not null, any area of high occupancy probability corresponding to this observation is only represented in the related slices of the grid (i.e. the slice corresponding to $c=[x, y, 0,0.8]$ in this case, see Fig. 3c).

It should be noticed that the cells located outside of the sensor field of view, or the cells "hidden" by one of the three sensor observations (i.e. the cells located behind the three detected obstacles) cannot be observed; consequently, nothing really consistent can be said about these cells, and the system has given an occupancy probability value of 0.5 for these cells.

Fig. 4 shows a sequence of successive prediction and estimation results given by the $B O F$. The experimental scenario involves a stationary obstacle and the Cycab moving forward at a velocity of $2.0 \mathrm{~m} / \mathrm{s}$. The obstacle is detected using the laser sensor; it finally goes out of the sensor field of view (see Fig. 4-d1), since the Cycab is moving forward. It should be noticed that the prediction step allows to infer knowledge about the current occupancy state of the cycab environment, even if the object is no longer observed by the sensor; this is the situation depicted by fig 4-d3, where an area of high occupancy probability still exists when the object is going out of the sensor field of view. In some sense, our prediction step can be seen as a "short-term memory", which allows to combine in an evolutive way past and current observations.

\section{E. BOF based collision avoidance}

In [1], we have shown how to avoid a mobile obstacle by combining the occupancy grid result given by the $B O F$, with a danger probability term computed using a "time to collision" criteria. In this approach, each cell $O_{x, y, v_{x}, v_{y}}^{k}$ of the grid is characterized by two probability distributions : the "occupancy" term $P\left(O_{x, y, v_{x}, v_{y}}^{k} \mid \mathcal{Z}^{k}\right)$ and the "danger" term $P\left(D_{x, y, v_{x}, v_{y}}^{k} \mid O_{x, y, v_{x}, v_{y}}^{k}\right)$. Using this model, it becomes possible to tune the velocity controls of the Cycab, according to the results of a combination of the two previous criteria.

This approach has experimentally been validated using the following scenario : the Cycab is moving forward; a pedestrian is moving from right to left, and during a small period of time the pedestrian is temporarily hidden by a parked car. Fig 5 shows some snapshots of the experiment : the Cycab brakes to avoid the pedestrian which is temporarily hidden by the parked car, then it accelerates as soon as the pedestrian has crossed the road.

\section{F. Discussion and performances}

Thanks to the previous assumptions, both the prediction step and the estimation step complexities increases linearly with the number of cells of the grid. This make the approach tractable in real situations involving reasonable grid sizes. This is the case for the experimental scenarios described in this section (e.g. size of the sensed space of $10 \mathrm{~m} \mathrm{~m}$ with a resolution of $0.5 \mathrm{~m}$, longitudinal velocity of $-3 \mathrm{~m} . \mathrm{s}^{-1}$ to $1 \mathrm{~m} . \mathrm{s}^{-1}$ with a resolution of $0.4 \mathrm{~m} . \mathrm{s}^{-1}$, lateral velocity of $-3.2 \mathrm{~m} . \mathrm{s}^{-1}$ to $3.2 \mathrm{~m} . \mathrm{s}^{-1}$ with a resolution of $0.4 \mathrm{~m} . \mathrm{s}^{-1}$ ). Using such a grid of 64.000 cells, the computation time for both prediction and estimation steps is about $100 \mathrm{~ms}$ on a $1 \mathrm{GHz}$ computer. This 


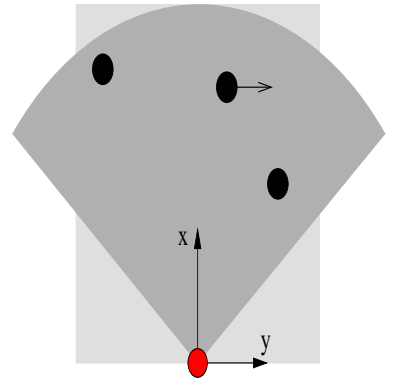

a)

$z_{1}=(8.3,-4,0,0)$

$z_{2}=(5,3,0,0)$

$z_{3}=(7.3,1.9,0,0.8)$

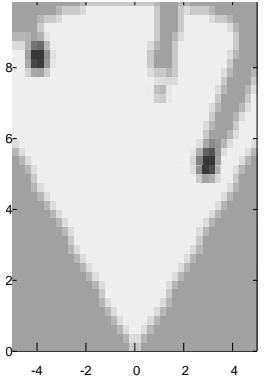

b)

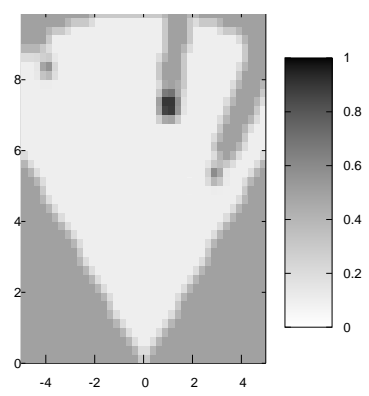

c)

$P\left(\left[O_{C}=o c c\right] \mid \mathcal{Z}\right)$

$[c=(x, y, 0,0.8)]$

Fig. 3. Example of a grid estimation using a single laser sensor located at $(0,0)$, in a scene including two stationary objects and an object moving from the left to the right at a velocity of $0.8 \mathrm{~m} / \mathrm{s}$. In this example, the robot is not moving. The occupancy probability value of each cell is represented by a gray level (see the colors correspondences on the right side of the fi gure). (a) The sensed environment and the three instantaneous sensor observations expressed in the $(x, y, \dot{x}, \dot{y})$ space. (b) Occupancy probability in a 2-dimensional slice of the grid corresponding to a null relative velocity $(i . e$. $c=[x, y, 0,0]$ ). (c) Occupancy probability in a 2-dimensional slice of the grid corresponding to a relative horizontal velocity 0.8 (i.e. $c=[x, y, 0,0.8]$ ).

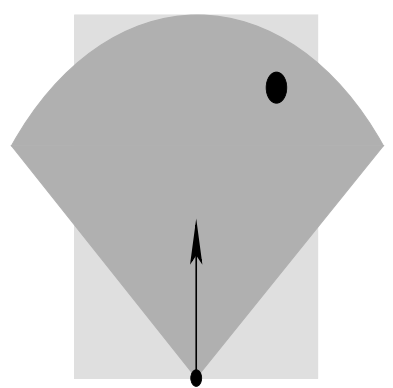

a.1.

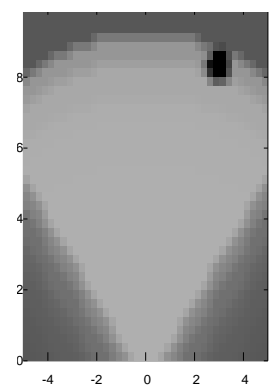

a.2.

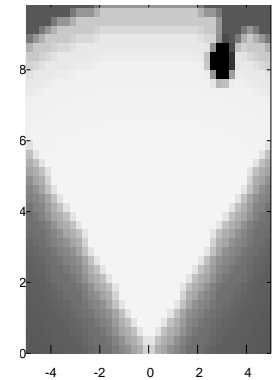

a.3.

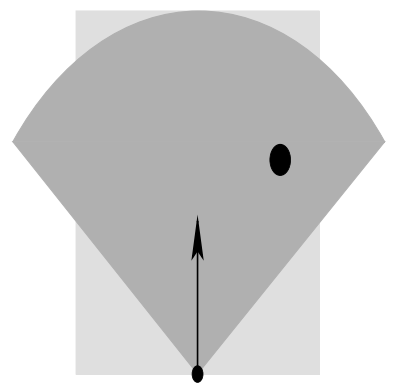

b.1.

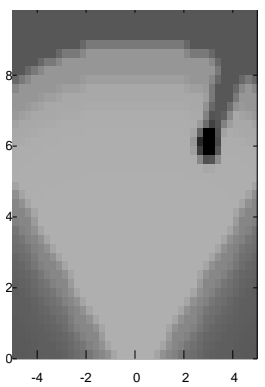

b.2.

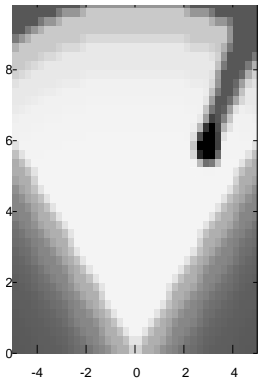

b.3.

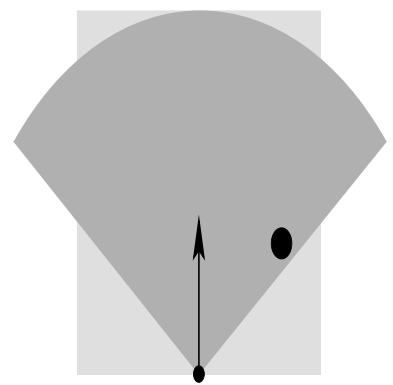

c.1.

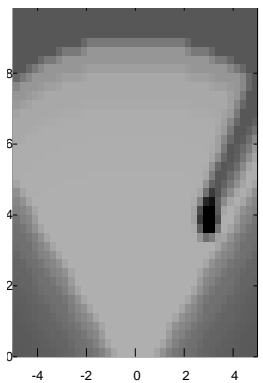

c. 2 .

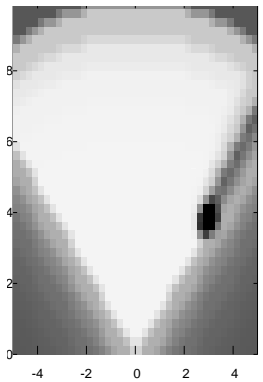

c.3.

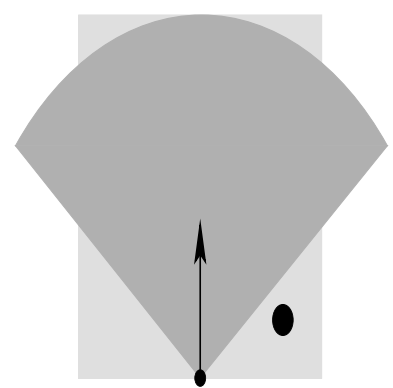

d.1.

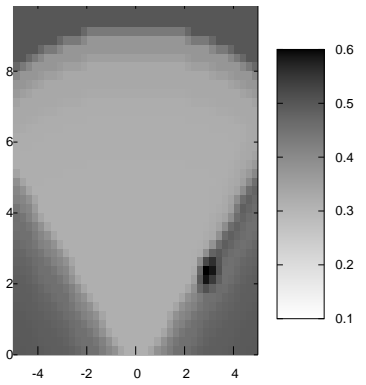

d. 2 .

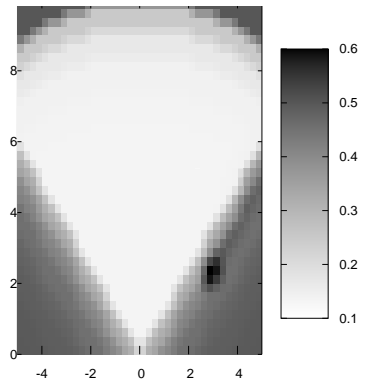

d.3.

Fig. 4. A short sequence of a dynamic scene involving a stationary obstacle and the Cycab moving forward at a velocity of $2.0 \mathrm{~m} / \mathrm{s}$. The second and the third row respectively show the results of the prediction and of the estimation steps of the $B O F$, in a 2-dimensional slice of the grid corresponding to a relative speed of $\dot{x}=-2.0$, i.e. $P\left(\left[O_{x y[\dot{x}=-2.0][\dot{y}=0.0]}^{t}=o c c\right]\right)$. 
recursive formulation :

$$
q^{t}=\frac{p\left(z^{t-1} \mid o c c\right)}{p\left(z^{t-1} \mid e m p\right)} q^{t-1}=q^{0} \prod_{i=0}^{t-1} \frac{p\left(z^{i} \mid o c c\right)}{p\left(z^{i} \mid e m p\right)}
$$

where $q_{t-1}$ is recursively evaluated.

Such updating operations are clearly non-linear. In order to be able to perform the update operations using only sums, we have decided to make use of a logarithmic form of the model :

$$
o d d s^{t}=o d d s^{0}+\sum_{i=0}^{t-1} \log \left(\frac{p\left(z^{i} \mid \text { occ }\right)}{p\left(z^{i} \mid e m p\right)}\right)
$$

where odds $s^{t}=\log \left(q^{t}\right)$.

Let $O b s\left(z^{t-1}\right)$ be the term $\log \left(\frac{p\left(z^{t-1} \mid o c c\right)}{p\left(z^{t-1} \mid e m p\right)}\right)$ in the eq. (10). $\operatorname{Obs}\left(z^{t-1}\right)$ represents the "observation" term of the update, and $o d d s(t-1)$ is the a priori term. Then, updating a $W O G$ can be done by adding the wavelet transform of observation terms to the wavelet representation of the map.

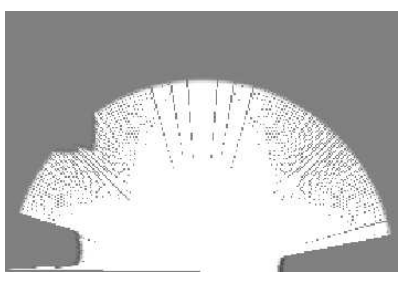

(a)

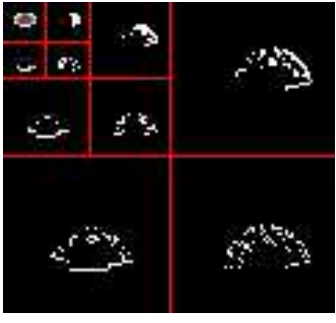

(b)
Fig. 6. Mapping obtained by a laser range-fi nder : (a) the obtained $O G$ model; (b) the related three first "detail spaces" of the WOG model and the complementary scaled space. The density of non-zero coeffi cients decreases drastically in wavelet space.

\section{Multi-scale semantic of logarithmic $O G$.}

In the case of the Haar wavelet basis (eq. 5), $\Phi_{s}^{(i, j)}$ has a constant value $(k)$ over the $\operatorname{domain}^{3}(s, i, j)$, and is equal to zero elsewhere. Then the integral of the scalar product is a sum over the cells enclosed in the domain $(s, i, j)$; this sum can be re-written as follows [16] :

$$
\begin{array}{r}
<\log \left(\frac{p^{t}(x, y)}{1-p^{t}(x, y)}\right) \mid \Phi^{s i j}> \\
=\int_{x, y \in \mathbb{R}^{2}} \log \left(\frac{p^{t}(x, y)}{1-p^{t}(x, y)}\right) \Phi^{s i j}(x, y) d_{x, y} \\
=k \log \left(\frac{\Pi_{c \in(s, i, j)} p\left(\left[O_{c}=o c c\right]\right)}{\Pi_{c \in(s, i, j)} p\left(\left[O_{c}=e m p\right]\right)}\right) \\
=k \log \left(q_{s}\right)
\end{array}
$$

where $c$ is the index of a cell in the square domain. $k \log \left(q_{s}\right)$ is the $\log$ of the ratio of two geometric means (cells are "all occupied" and cells are "all empty") which leads us to

\footnotetext{
${ }^{3}$ With value: $k=2^{-s}$
}

a continuous semantics. Let define full as the event "every subcell is occupied" then:

$$
\Pi_{c \in(s, i, j)} p\left(\left[O_{c}=o c c\right]\right)=p\left(\bigwedge_{c \in(s, i, j)}\left[O_{c}=o c c\right]\right)=p(f u l l)
$$

Let define open as the event "every subcell is empty".

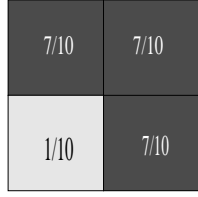

(a)

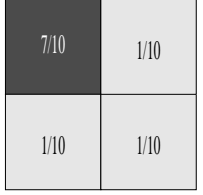

(b)

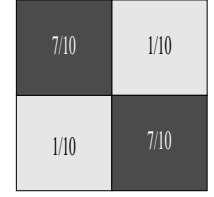

(c)
Fig. 7. Three confi gurations of a subsquare domain at scale 1/2: the probability that the square domain 7(a) is fully "occupied" is 0.0343 ; its probability of being fully "empty" is 0.0243 . The occupancy ratio related to the "occupied" and "empty" properties is $P($ open $\mid$ open $\vee$ full $)=0.58$; this means that the related square domain is considered as "occupied". The occupancy ratio in 7 (b) is 0.003 ; it is of 0.06 in 7 (c).

$q_{s}$ leads immediately to the conditional probabilities:

$$
\begin{aligned}
P(\text { open } \mid \text { open } \vee \text { full }) & =q_{s} /\left(1+q_{s}\right) \\
P(\text { full } \mid \text { open } \vee \text { full }) & =1 /\left(1+q_{s}\right)
\end{aligned}
$$

which express a continuous ratio between the two events full and open (Fig. 7).

The multi-scale information which can be computed by this approach, is directly derived from these two basic cases. Consequently, only 2 relevant events can be considered for a square domain $(s, i, j)$ containing $n$ subcells (i.e. where $2^{n}$ possibilities can be defined by the binary vector representing the possible occupancy of these $n$ subcells), Fig. 8(c) illustrates. Fig. 7 shows the information which can be derived from the previous multi-scale occupancy model. The next step will be to exploit this concise information for elaborating multiscale algorithms.

(iii) WOG implementation and experimental results.

This approach has been implemented and tested on the Cycab equipped with a laser sensor an with our SLAM method [17]. In the experiments, the size of the finer scale is equal to $6.25 \mathrm{~cm}$, and we have used 5 different scales (where the size is multiplied by 2 for each new scale); thus, the size of the coarsest cells is equal to $1 \mathrm{~m}$. The size of the square window which is used for constructing the WOG model, is chosen in order to contain the whole sensor field of view. The content of this window is updated at each laser scan (see Fig. 6; then, the Haar wavelet transform is applied to this sensory data, and incrementally added to the current $W O G$ model. The chosen compression threshold is a compromise between data fitting and sparsity (wavelet terms which are lower than the threshold are removed).

Fig. 8 shows the results obtained on the car park of INRIA. These experimental results clearly shows that we have obtained a significant reduction of the size of the model (about $80 \%$ relatively to the $O G$ model), and that the interesting details are still represented (such as the beacons represented by 


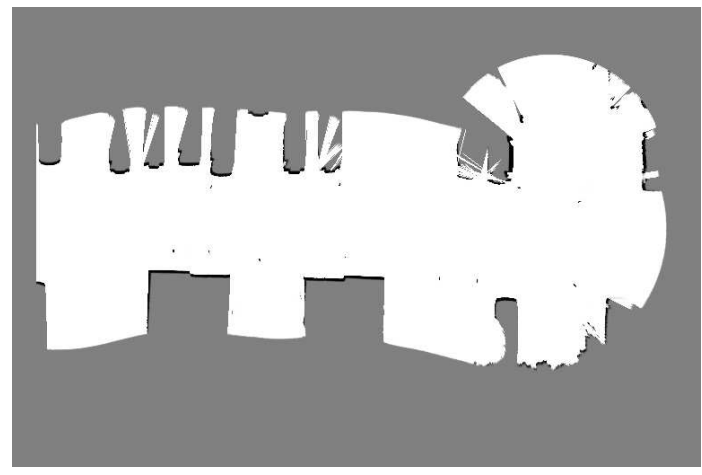

(a)

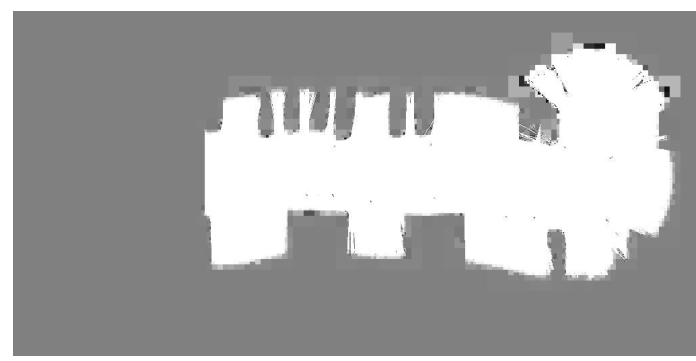

(b)

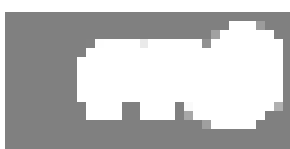

(c)

Fig. 8. Maps of the car park of INRIA. (a) The $O G$ model contains 393,126 cells, while the $W O G$ model contains 78,742 cells. (b) The $O G$ model reconstructed from the previous $W O G$ model; it can be seen that significant details such as beacons have been captured by the $W O G$ representation (the shapes of maps (a) and (b) are very close). (c) The empty space is pretty well approximated at the coarser scale.

dark dots in Fig. 8(b)). It should be noticed that the coarser model give a quite good representation of the empty space (see Fig. 8(c)); this model could be used for path planning, and refined when necessary using the wavelet model. In the previous experiments, the map building has been done in realtime.

\section{MEDIUM-TERM MOTION PREDICTION}

Predicting the motion of the objects moving in a given environment is a key to safe navigation. The purpose of this section is to present a prediction scheme well adapted to the target application. The approach focuses on motion prediction for objects which are able to execute trajectories as a result of an internal motion planning process or decision mechanism (eg persons, animals and robots). It is assumed that such plans are made with the intention to reach a specific goal, thus the name intentional motion which will be used hereafter to designate this kind of motion.

Assuming that both the motion planning algorithm and the internal state of a moving object are known, predicting its trajectory simply consists in replicating the planning process in order to find the intended trajectory. However, this assumption is hard to meet in real situations. Neither the planning model nor the internal state are known or observable (what is the motion planning algorithm of a human being?) and they must be inferred from observed motion before performing prediction. From our point of view, a complete intentional motion prediction approach should address the following problems:

Plan modelling: Considerable research effort has been dedicated to finding mathematical motion models which are at the same time expressive (ie able to model complex plans) and computationally efficient (ie able to work in real time). One of the first example in the literature is [18], [19] which models whole plans as typical geometric paths represented by sequences of linear segments. The work of [20] and [21] represents plans as typical trajectories (ie time-series of states). In [22] a hierarchic Hidden Markov Model known as Abstract Hidden Markov Model (AHMM) is used to represent all the possible plans for a given environment.
Parameter learning: Plan model parameters should be adjusted to work with particular problem instances (eg cars moving in a parking lot, people in a shopping mall, etc.). Automatic learning of these parameters constitutes a very active and challenging research area. For example, ExpectationMaximisation is applied to cluster trajectories in [20] and to find AHMM parameters in [23]. In [21], a general scheme is proposed on the basis of pairwise clustering algorithms.

Plan recognition: Having an instantiated plan model is not enough to perform prediction, it is necessary to recognise the actual plan being executed from the observations gathered through sensors. Ref. [21] use a distance criterion. AHMMs [22] perform recognition by recursively integrating a belief of the plan that the object is executing.

\section{A. Proposed Approach}

Before describing our approach and explaining how it addresses the problems mentioned above, we will describe the expected input and output data in the context of the target application:

The input of the learning algorithm is a continuous stream of observations $o_{t}=\left\{o_{1}, o_{2}, \cdots\right\}$ gathered through a sensor (eg the parking vision system). In order to keep notation simple, we will assume that no more than one object is observed at the same time, noting that the approach is easily generalisable to the multi-object case.

Every observation $o_{t}=\left(x_{t}, y_{t}, \eta_{t}\right)$ consists of the estimates of the object's position ${ }^{4}$ at time $t$ as returned by the sensor and a binary variable $\eta_{t}$ which indicates whether the object has reached the end of its trajectory $(\eta=1)$ or not $(\eta=0)$. A trajectory ends when the obstacle stops moving or exits the environment.

Predictions will be output in the form of a conditional probability distribution $p\left(q_{t+k} \mid o_{1: t}\right)$ ie the expected state of the object $k$ time steps into the future given all the observed data up to the present moment.

${ }^{4}$ Full state observations (ie $\left(x_{t}, y_{t}, x_{t}^{\prime}, y_{t}^{\prime}\right)$ ) may also be used as input by the algorithm. 
top of figure 10a). Fig. 10b presents the state of the GNG structure after processing 1000 trajectories.

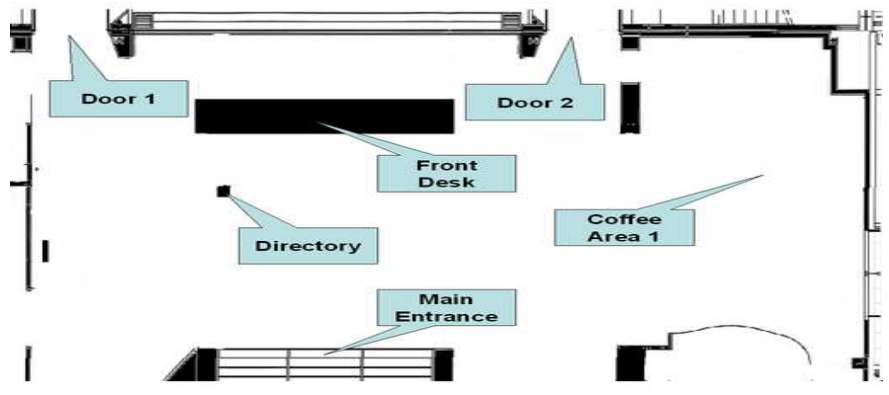

a) Test environment

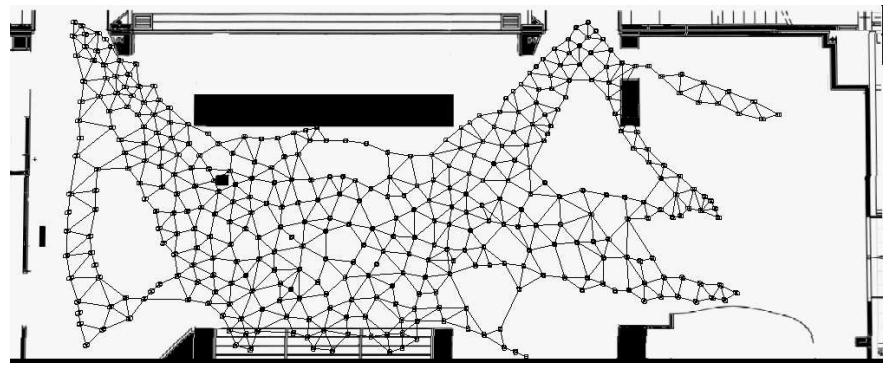

b) Resulting GNG network

Fig. 10. Using GNG to represent discrete states in the laboratory entry hall.

2) Goals Learning: The problem of automatically identifying the goals that an object seeks to reach using only observation data is very difficult since these goals are often related to information which is not present in this data (eg the existence of a door or a bulletin board).

The approach taken here aims to identify goals based on a simple hypothesis: when an object stops moving is because it has reached its goal. This leads to a simple goal identification scheme: every observation $o_{t}$ having $\eta_{t}=1$ is sent to a GNG structure which clusters this information together into high level goals.

The nodes of the resulting GNG graph corresponds to goals ${ }^{5}$. The graph itself may be used to identify the goal that correspond to a given end-state observation:

$$
\gamma\left(o_{t}\right)=\min \arg _{i}\left\|\left(x_{t}, y_{t}\right)-\mu_{i}^{g}\right\|
$$

In the case of observations having $\eta_{t}=0$ its goal is not known when the observation is received, it is necessary to wait until an end-state observation is received in order to perform the association and propagate this information to previous observation data. This means that a temporary data structure (eg a stack) has to be used in order to keep the "unlabelled" observations.

3) Transition Model Learning: Transition probabilities are coded in a three dimensional matrix $A$ where every cell $A[i, j, k]$ represents the number of times that a transition from state $j$ to state $k$ has been observed knowing that the intended

\footnotetext{
${ }^{5}$ notations $\mu_{i}^{s}$ and $\mu_{i}^{g}$ will be used henceforth in order to distinguish between state and goal GNG's
}

goal is state $k$. This leads to the following expression of the probability:

$$
\begin{aligned}
& p\left(\left[q_{t}=j\right] \mid[\gamma=g],\left[q_{t-1}=i\right]\right)= \\
& \quad \frac{\mathcal{A}[g, i, j]}{\sum_{h} \mathcal{A}[g, i, h]}
\end{aligned}
$$

Learning is performed on a maximum likelihood criterion. At each time step, the most likely present state $q_{t}$ is estimated in order to update matrix $A$.

For every received observation, the intended goal $\gamma=g$ is found using Eq. 17. Then the Viterbi algorithm is used in order to find $q_{t}$ given the past state $q_{t-1}=i$ (which has been estimated in the previous iteration) and the current observation $o_{t}$ :

$$
\begin{aligned}
q_{t}= & \max \arg _{j}\{ \\
& p\left(\left[q_{t}=j\right] \mid[\gamma=g],\left[q_{t-1}=i\right]\right) \\
& \left.p\left(o_{t} \mid\left[q_{t}=j\right]\right)\right\}
\end{aligned}
$$

Updating the matrix $A$ is straightforward knowing $p, i$ and $j: A[p, i, j] \leftarrow A[p, i, j]+1$.

This update only takes place when when the current observation does not correspond to the first step of a plan (ie no transition has been observed and $\eta_{t-1} \neq 1$ ). If this is the case, only the current state is estimated using the following expression:

$$
q_{t}=\max \arg _{i}\left\{p\left(o_{t} \mid\left[q_{t}=i\right]\right)\right\}
$$

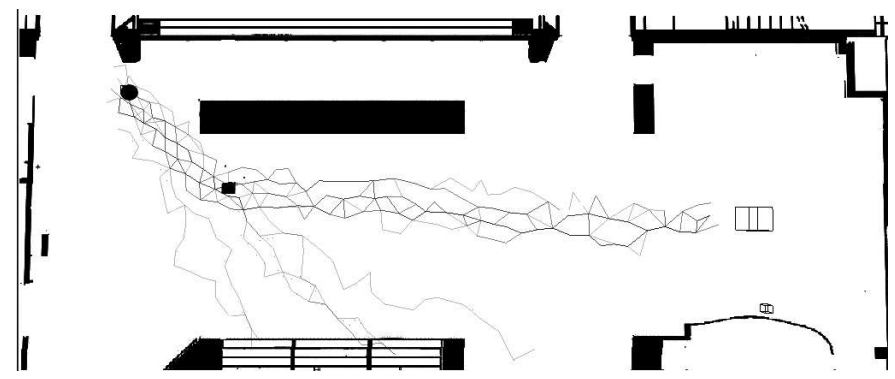

Fig. 11. Motion prediction

4) Intentional Motion Prediction: As mentioned earlier, the prediction of the future motion of a given moving object is given by the conditional probability distribution $p\left(q_{t+k} \mid o_{1: t}\right)$ $i e$ the expected state of the object $k$ time steps into the future given all the observed data up to the present moment.

In order to estimate the probability of the plan that an object is executing as well as its current state, we have used a particle filter with a resampling step to integrate new observations [30]. Alternatively, an exact inference mechanism like the junction tree algorithm [31] may be used. Fig. 11 gives an example of most likely future motions for a moving object currently located in the upper-left part of the environment. 


\section{SAFE NAVIGATION In DYNAMIC EnVIRONMENTS}

When placed in a dynamic environment, an autonomous system must consider the real time constraint that such an environment imposes. Indeed, the system has a limited time only to make a decision about its future course of action otherwise it might be in danger by the sole fact of being passive. The time available depends upon a factor that we will call the dynamicity of the environment and which is a function of the system and the moving objects'dynamics.

In this context, basing the decision making process on a motion planning technique ${ }^{6}$ leaves little hope to fulfil this real-time constraint given the intrinsic time complexity of the motion planning problem [32] (even if using randomised approaches). This certainly explain why so many reactive methods ${ }^{7}$ have been developed in the past (cf [33], [34], [35], [36], [37], [38], [39], [40], [41], [42] or [43]. However, reactive approaches are confronted with two key issues: the convergence and the safety issues. As for convergence, their lack of lookahead may prevent the system to ever reach its goal. As for safety, what guarantee is there that the system will never find itself in a dangerous situation eventually yielding to a collision?

Partial Motion Planning (PMP) is the answer we propose to the problem of navigation in dynamic environments. It is especially designed in order to take into account the realtime constraint mentioned above. PMP is a motion planning scheme with an anytime flavor: when the time available is over, PMP returns the best partial motion to the goal computed so far. Like reactive scheme, PMP is also confronted to the convergence and safety issues. At this point, we have decided to focus on the safety issue and to propose a solution relying upon the the concept of Inevitable Collision States (ICS) originally introduced in [44]. An ICS is a state such that no matter what the future motion of the system is, it eventually collides with an obstacle. ICS takes into account the dynamics of both the system and the moving obstacles. By computing ICS-free partial motion, the system safety can be guaranteed.

PMP is detailed in section IV-A while section IV-B presents the ICS concept. Finally, an application of PMP to the case of a car-like system in a dynamic environment is presented in section IV-C.

\section{A. Partial Motion Planning}

As mentioned earlier, a robotic system cannot in general safely stand still in a dynamic environment (it might be collided by a moving obstacle). It has to plan a motion within a bounded time and then execute it in order to remain safe. The time $\delta_{c}$ available to calculate a new motion is function of the nature and dynamicity of the environment. To take into account the real-time constraint that stems from a dynamic environment, we propose a scheme that calculates partial motions only according to the the following cycle (also depicted in Fig. 12):

\footnotetext{
${ }^{6}$ Wherein a complete motion to the goal is computed a priori.

${ }^{7}$ Wherein only the next action is determined at each time step.
}

PMP Algorithm

Step1: $\quad$ Get model of the future

Step2: Build tree of partial motions towards the goal

Step3: When $\delta_{c}$ is over, return best partial motion

Step4: $\quad$ Repeat until goal is reached

Like motion planning, partial motion planning requires a model of the environment, the first step is aimed at getting this model. The required model is provided by the environment modelling and motion prediction functions presented earlier. The periodic iterative PMP scheme proposed in this paper accounts for both the planning time constraints and the validity duration of the predictions made.

\section{B. Inevitable Collision States}

Like every method that computes partial motion only, PMP has to face a safety issue: since PMP has no control over the duration of the partial trajectory that is computed, what guarantee do we have that the $\operatorname{robot} \mathcal{A}$ will never end up in a critical situation yielding an inevitable collision? As per [44], an Inevitable Collision State (ICS) is defined as a state $s$ for which no matter the control applied to the system is, there is no trajectory for which the system can avoid a collision in the future. The answer we propose to the safety problem lies then in the very fact that the partial trajectory that is computed is ICS-free. Meaning that, even in the worst case scenario where the duration $\delta_{h_{i}}$ of the partial trajectory is shorter than the cycle time $\delta_{c}, \mathcal{A}$ can always execute one of the existing safe trajectory. The overall safety is guaranteed as long as the initial state is ICS-free (which is something that can be reasonably assumed). Now, determining whether a given state of $\mathcal{A}$ is an ICS or not is a complex task since it requires to consider all possible future trajectories for $\mathcal{A}$. However, it is possible to take advantage of the approximation property demonstrated in [44] in order to compute a conservative approximation of the set of ICS. This is done by considering only a subset $\mathcal{I}$ of the full set of possible future trajectories.

\section{Case Study}

In this section we present the application of PMP to the case of a car-like vehicle $\mathcal{A}$ moving on a planar surface $\mathcal{W}$ and within a fully observable environment cluttered with stationary and dynamic obstacles. A control of $\mathcal{A}$ is defined by the couple $(\alpha, \gamma)$ where $\alpha$ is the rear wheel linear acceleration. and $\gamma$ the steering velocity. The motion of $\mathcal{A}$ is governed by the following differential equations:

$$
\left[\begin{array}{c}
\dot{x} \\
\dot{y} \\
\dot{\theta} \\
\dot{v}_{r} \\
\dot{\xi}
\end{array}\right]=\left[\begin{array}{c}
v_{r} \cos \theta \\
v_{r} \sin \theta \\
\frac{\tan \xi v_{r}}{L} \\
0 \\
0
\end{array}\right]+\left[\begin{array}{l}
0 \\
0 \\
0 \\
1 \\
0
\end{array}\right] \alpha+\left[\begin{array}{l}
0 \\
0 \\
0 \\
0 \\
1
\end{array}\right] \gamma
$$

with $\alpha \in\left[\alpha_{\min }, \alpha_{\max }\right]$ (acceleration bounds), $\gamma \in$ $\left[\gamma_{\min }, \gamma_{\max }\right]$ (steering velocity bounds), and $|\xi| \leq \xi_{\max }$ (steering angle bounds). $L$ is the wheelbase of $\mathcal{A}$.

For practical reasons, the duration of the trajectories of $\mathcal{I}$ has to be limited to a given time horizon that determines the 


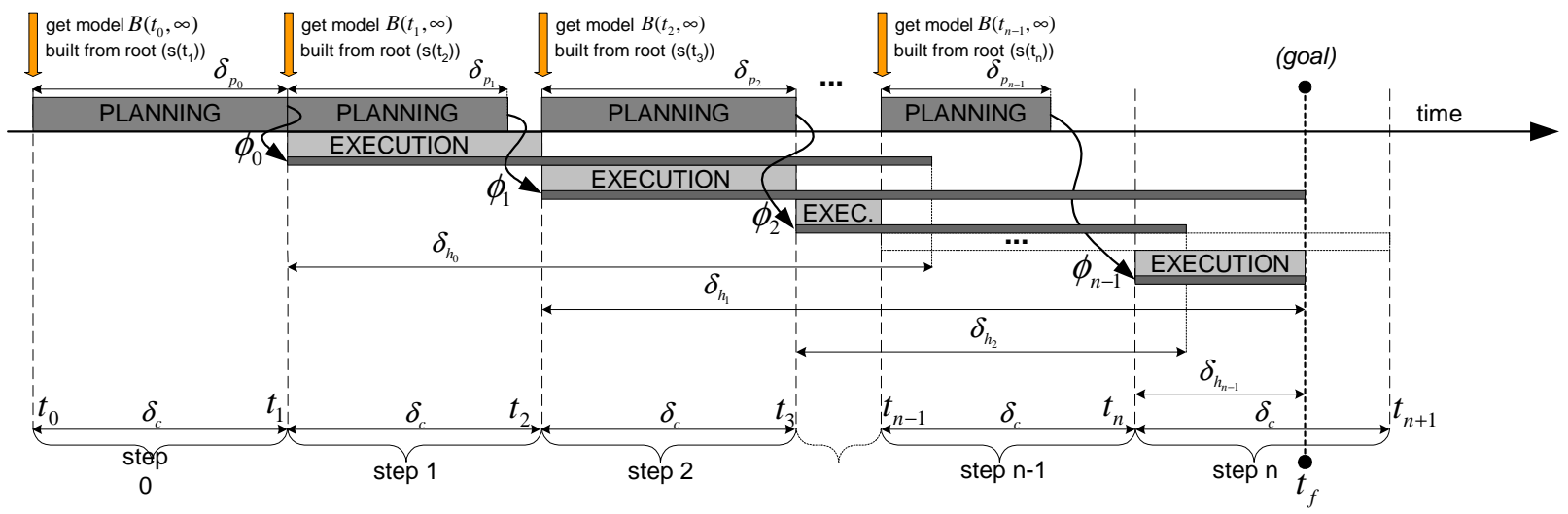

Fig. 12. Partial Motion Planning iterative cycle

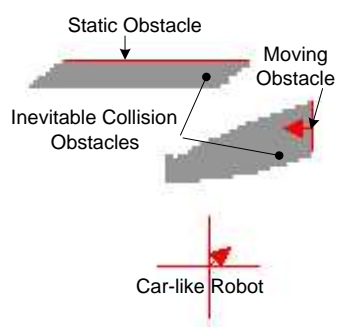

(a) $(\theta, v)=(0.9,1.0)$

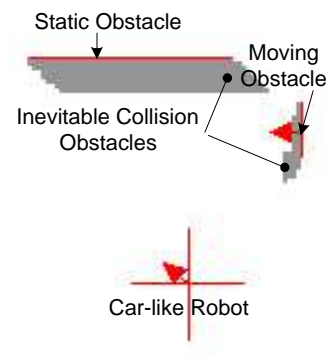

(c) $(\theta, v)=(2.2,1.0)$

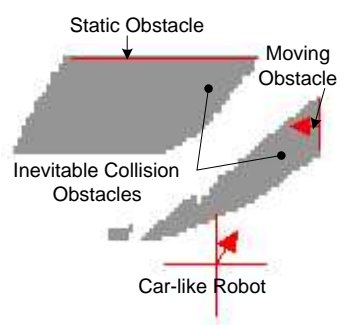

(b) $(\theta, v)=(0.9,2.0)$

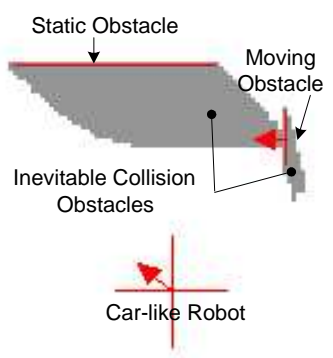

(d) $(\theta, v)=(2.2,2.0)$

Fig. 13. $(\theta, v)$-slices of the state space of $\mathcal{A}$. Shaded regions are ICS respectively defined for the braking trajectory of control $\left(\alpha_{\text {min }}, \dot{\xi}_{\text {min }}\right)$ (top), and all braking trajectories with controls selected from $\left[\left(\alpha_{\min }, \dot{\xi}_{\max }\right),\left(\alpha_{\min }, 0\right),\left(\alpha_{\min }, \dot{\xi}_{\min }\right)\right]$ (bottom).

overall level of safety of $\mathcal{A}$. In our case, the subset $\mathcal{I}$ considered in order to compute a conservative approximation of the set of ICS includes the braking trajectories with a constant control selected from $\left[\left(\alpha_{m i n}, \dot{\xi}_{m a x}\right),\left(\alpha_{m i n}, 0\right),\left(\alpha_{m i n}, \dot{\xi}_{m i n}\right)\right]$, and applied over the time necessary for $\mathcal{A}$ to stop.

Fig. 13 depicts the ICS obtained when different set of braking trajectories are considered. Each subfigure represents a $(\theta, v)$-slice of the full $5 \mathrm{D}$ state space of $\mathcal{A}$. In the top subfigures, only the braking trajectory of control $\left(\alpha_{\min }, \dot{\xi}_{m i n}\right)$ is considered. In the bottom subfigures, the three braking trajectories are considered.

In PMP, checking whether a state is an ICS or not is carried

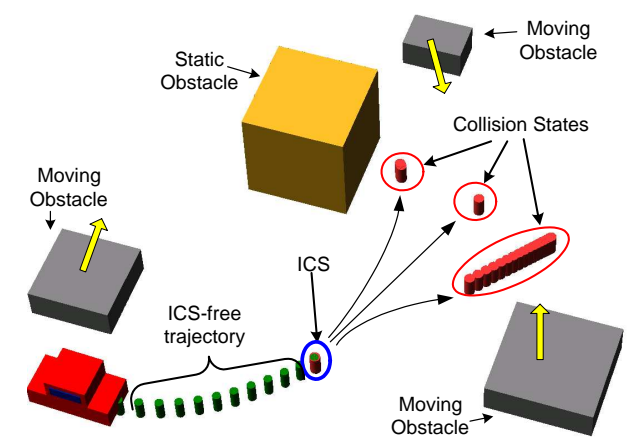

Fig. 14. The state labelled ICS is an ICS since the three braking trajectories issued from it yield collisions.

out by testing if all the braking trajectories yield a collision with one of the moving obstacles. If so, the state is an ICS. In fig. 14), the collision states in red represent the collision that will occur in the future from this state for all trajectories of $\mathcal{I}$. In this case, since all trajectories collide in the future, this state is an ICS. In PMP, every new state is similarly checked to be an ICS or not over $\mathcal{I}$. In case all the trajectories appear to be in collision in the future, this state is an ICS and is not selected.

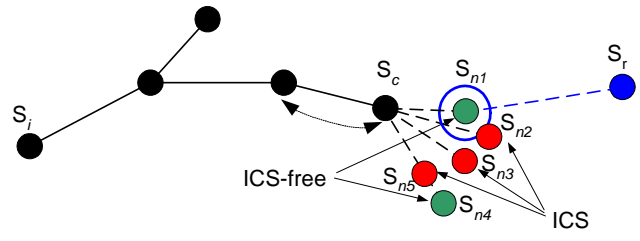

Fig. 15. Search tree construction principle.

The exploration method used is the well known Rapidly-Exploring Random Tree method (RRT) [45]. RRT incrementally builds a tree in the state space of $\mathcal{A}$. The basic principle of RRT is depicted in Fig. 15. A state $s_{r}$ is randomly selected first. Then, the closest node in the tree, say $s_{c}$, is determined. Constant controls selected from $\mathcal{U}_{2 D}=\left[\left(\alpha_{\max }, 0\right) ; \quad\left(\alpha_{\max }, \dot{\xi}_{\text {max }}\right) ; \quad\left(\alpha_{\text {max }}, \dot{\xi}_{\text {min }}\right) ;\left(0, \dot{\xi}_{\text {max }}\right) ;\right.$ $\left.(0,0) ; \quad\left(0, \dot{\xi}_{\text {min }}\right) ;\left(\alpha_{\text {min }}, \dot{\xi}_{\text {max }}\right) ; \quad\left(\alpha_{\text {min }}, 0\right) ; \quad\left(\alpha_{\text {min }}, \dot{\xi}_{\text {min }}\right)\right]$ are then applied to $s_{c}$ for a duration $\epsilon$, they yield a set 


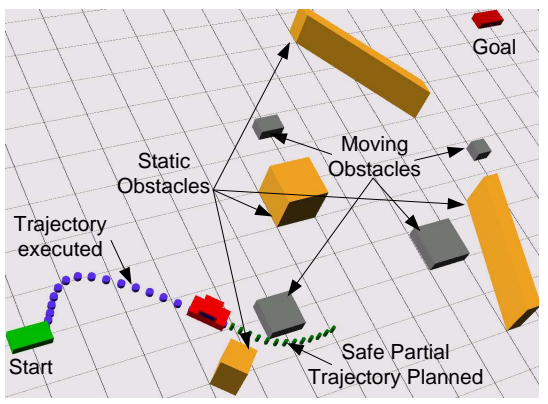

(a) Avoidance of the fi rst moving obstacle

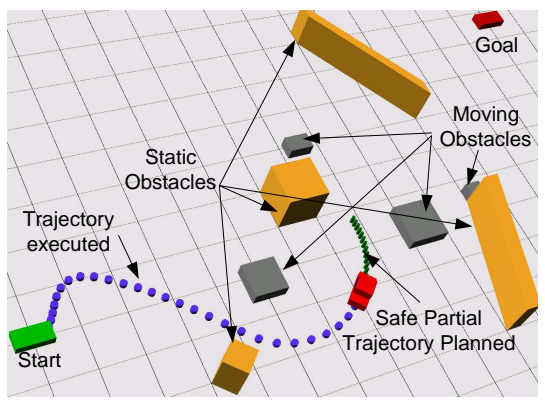

(b) Braking in front of unavoidable moving obstacles

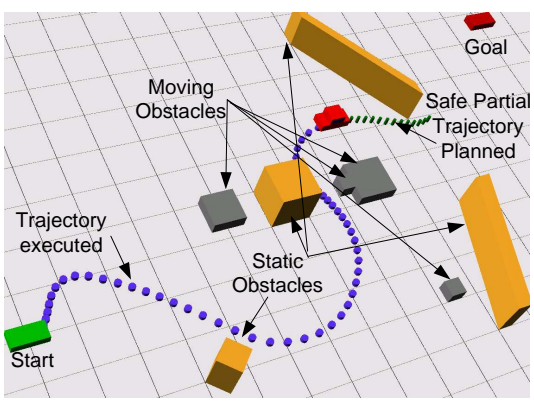

(c) Avoidance of the last static obstacle

Fig. 16. Results of a 2D safe Partial Motion Planning $\left(\delta_{c}=1 \mathrm{~s}, v_{\max }=2.0 \mathrm{~m} / \mathrm{s}, \xi_{\max }=\pi / 3 \mathrm{rad}, \dot{\xi}_{\max }=0.2 \mathrm{rad} / \mathrm{s}, \alpha_{\max }=0.1 \mathrm{~m} / \mathrm{s}^{2}\right)$

of candidate trajectories ending in given states $s_{n i}$. These candidate trajectories are pruned out: only are kept the trajectories that are collision-free and whose final state is ICS-free (as per property 2, such trajectories are ICS-free). Finally, the trajectory whose final state is closer to $s_{r}$ is selected and added up to the tree. This process is repeated until the end of the time available where the best partial trajectory extracted from the tree is returned.

In Fig. 16 we can see an example of a navigation from a still starting state (green box) to a still goal state (red box). The environment is cluttered with moving (grey) and static (orange) obstacles. In 16(a) one can observe how the safe partial trajectory (green) is calculated and planned within the time-state space in order to avoid the obstacle moving upward. The states in blue behind the car, is the trajectory, built from partial trajectories from the previous PMP cycles and (ideally) executed by the robot. In 16(b) we can observe that the car was obliged to slow down at the intersection of several obstacles, since no other safe trajectories could be found, before to reaccelerate. In 16(c) the system has planned a partial trajectory that avoids the last static obstacle.

\section{CONCLUSION}

This paper addressed the problem of navigating safely in a open and dynamic environment sensed using both onboard and external sensors. After a short presentation of the context and of the related open problems, we focused on two complementary questions: how to interpret and to predict the motions and the behaviors of the sensed moving entities ? how to take appropriate goal-oriented navigation decisions in such a rapidly changing and sensed environment ?

In order to answer these questions, we have proposed an approach including three main complementary functions: (1) Scene interpretation and short-term motion prediction for the sensed potential obstacles, using the "Bayesian Occupancy Filtering" approach (BOF) (2) Medium-term motion and behavior prediction for the observed entities, using motion pattern learning and hierarchical Hidden Markov Models; (3) On-line goal-oriented navigation decision in a dynamic environment, using the "Partial Motion Planning" paradigm $(P M P)$.
The first function $(B O F)$ has experimentally been validated on our experimental vehicle (the Cycab), for avoiding partially observed moving obstacles. A scenario involving the Cycab, a moving pedestrian, and a parked car which temporarily hide the pedestrian to the sensors of the Cycab, has successfully been executed. In this experiment, the avoidance behavior has been obtained by combining the occupancy probability and the danger probability of each cell of the grid. The second function has experimentally been validated on some indoor data (the INRIA entry hall), using about 1000 tracked human trajectories for the initial learning phase. At the moment, the last function $(P M P)$ has only been experimented in simulation.

Current work mainly deals with three major points: (1) Improvement of the prediction approaches for making it possible to cope with larger environments (such as complex urban traffic situations), while preserving the efficiency property; the current development on the WOG model is an example of this work. (2) Fusion of our current interpretation and prediction paradigms with higher-level information (e.g. GPS maps, moving entities properties, nominal behaviors ...) to better estimate the scene participants behaviors. (3) Integration of the three previous functions, and implementation and test this new navigation system on our experimental platform involving the INRIA car park, several Cycabs, and both inboard and infrastructure sensors.

Acknowledgements.: This work has been partially supported by several national and international projects and funding bodies: European projects Carsense, CyberCars \& PreVent/ProFusion; French Predit projects Puvame \& MobiVip. The authors would like to thanks Pierre Bessière, Christophe Coué and Cedric Pradalier for their contributions and fruitful discussions.

\section{REFERENCES}

[1] C. Coué, C. Pradalier, and Laugier C. Bayesian programming for multi-target tracking: an automotive application. In Proceedings of the International Conference on Field and Service Robotics, Lake Yamanaka (JP), July 2003.

[2] H.P. Moravec. Sensor fusion in certainty grids for mobile robots. AI Magazine, 9(2), 1988. 
[3] A. Elfes. Using occupancy grids for mobile robot perception and navigation. IEEE Computer, Special Issue on Autonomous Intelligent Machines, Juin 1989.

[4] S. Thrun. Robotic mapping: A survey. In Exploring Artificial Intelligence in the New Millenium. Morgan Kaufmann, 2002.

[5] E. Prassler, J. Scholz, and A. Elfes. Tracking multiple moving objects for real-time robot navigation. Autonomous Robots, 8(2), 2000.

[6] O. Lebeltel. Programmation Bayésienne des Robots. Thèse de doctorat, Institut National Polytechnique de Grenoble, Grenoble, France, Septembre 1999.

[7] O. Lebeltel, P. Bessière, J. Diard, and E. Mazer. Bayesian robot programming. Autonomous Robots, 16:49-79, 2004.

[8] C. Coué and P. Bessière. Chasing an elusive target with a mobile robot. In Proceedings of the IEEE-RSJ International Conference on Intelligent Robots and Systems, Hawai (HI), 2001.

[9] A. H. Jazwinsky. Stochastic Processes and Filtering Theory. New York : Academic Press, 1970.

[10] R.E. Kalman. A new approach to linear filtering and prediction problems. Journal of basic Engineering, 35, Mars 1960.

[11] G. Welch and G. Bishop. An introduction to the Kalman fi lter. available at http://www.cs.unc.edu/ welch/kalman/index.html.

[12] I. Daubechies. Ten Lectures on Wavelets. Number 61 in CBMS-NSF Series in Applied Mathematics. SIAM Publications, Philadelphia, 1992.

[13] Stéphane Mallat. A Wavelet Tour of Signal Processing. Academic Press, San Diego, 1998

[14] D. K. Pai and L.-M. Reissell. Multiresolution rough terrain motion planning. In IEEE Transactions on Robotics and Automation, volume 1, pages 19-33, February 1998.

[15] Bruno Sinopoli, Mario Micheli, Gianluca Donato, and T. John Koo. Vision based navigation for an unmanned aerial vehicle. In Proceedings of the International Conference on Robotics and Automation, May 2001.

[16] Manuel Yguel, Olivier Aycard, and Christian Laugier. Internal report: Wavelet occupancy grids: a method for compact map building. Technical report, INRIA, 2005.

[17] C. Pradalier and S. Sekhavat. Simultaneous localization and mapping using the geometric projection filter and correspondence graph matching. Advanced Robotics, 2004. To appear.

[18] E. Kruse, R. Gutsche, and F.M. Wahl. Estimation of collision probabilities in dynamic environments for path planning with minimum collision probability. In Proceedings of IROS, pages 1288-1295, 1996.

[19] E. Kruse, R. Gusche, and F. M. Wahl. Acquisition of statistical motion patterns in dynamic environments and their application to mobile robot motion planning. In Proceedings of the IEEE/RSJ Int. Conf. on Intelligent Robots and Systems, pages 713-717, Grenoble, France, 1997.

[20] Maren Bennewitz, Wolfram Burgard, and Sebastian Thrun. Learning motion patterns of persons for mobile service robots. In Proceedings of the IEEE Int. Conf. On Robotics and Automation, pages 3601-3606, Washington, USA, 2002

[21] Dizan Vasquez and Thierry Fraichard. Motion prediction for moving objects: a statistical approach. In Proc. of the IEEE Int. Conf. on Robotics and Automation, pages 3931-3936, New Orleans, LA (US), April 2004

[22] H. Bui, S. Venkatesh, and G. West. Policy recognition in the abstract hidden markov models. Journal of Artificial Intelligence Research, 17:451-499, 2002

[23] Sarah Osentoski, Victoria Manfredi, and Sridhar Mahadevan. Learning hierarchical models of activity. In IEEE/RSJ International Conference on Intelligent Robots and Systems, Sendai, Japan, 2004.

[24] Y. Linde, A. Buzo, and R.M. Gray. An algorithm for vector quantizer design. IEEE Transactions on Communications, COM-28:84-95, 1980.

[25] Bernd Fritzke. A growing neural gas network learns topologies. Advances in Neural Information Processing Systems, 1995.

[26] Teuvo Kohonen. Self-Organizing Maps, volume 30 of Springer Series in Information Sciences. Springer, Berlin, Heidelberg, 1995. (Second Extended Edition 1997).

[27] M. Martinetz and K. J. Schulten. A "neural-gas" network learns topologies. In T. Kohonen, K. Makisara, O. Simula, and editors J. Kangas, editors, Proceedings of International Conference on Articial Neural Networks, volume I, pages 397-402, North-Holland, Amsterdam, 1991

[28] G.A. Carpenter, S. Grossberg, and D.B. Rosen. Fuzzy art: An adaptive resonance algorithm for rapid, stable classifi cation of analog patterns. In Proc. Int. Joint Conf. Neural Networks, volume II, pages 411-420, Seattle, USA, 1991.

[29] Antonin Guttman. R-trees: A dynamic index structure for spatial searching. In SIGMOD Conference, pages 47-57, Boston MA (USA), 1984. on particle fi lters for on-line non-linear/non-gaussian bayesian tracking. IEEE Transactions on Signal Processing, 50(2):174-188, February 2002.

[31] C. Huang and A. Darwiche. Inference in belief networks: A procedural guide. International Journal of Approximate Reasoning, 15(3):225-263, 1996.

[32] J. H. Reif and M. Sharir. Motion planning in the presence of moving obstacles. In Proc. of the IEEE Symp. on the Foundations of Computer Science, pages 144-154, Portland, OR (US), October 1985.

[33] R.C. Arkin. Motor schema-based mobile robot navigation. International Journal of Robotics Research, 8(4):92-112, August 1989.

[34] J. Borenstein and Y. Koren. The vector fi eld histogram - fast obstacle avoidance for mobile robots. IEEE Journal of Robotics and Automation, 7(3):278-288, June 1991.

[35] R. Simmons and S. Koenig. Probabilistic robot navigation in partially observable environments. In Proceedings of the International Joint Conference on Artificial Intelligence, pages 1080-1087, 1995.

[36] D. Fox, W. Burgard, and S. Thrun. The dynamic window approach to collision avoidance. Technical Report IAI-TR-95-13, 11995.

[37] M. Khatib. Sensor-based motion control for mobile robots. $\mathrm{PhD}$ thesis, LAAS-CNRS December, 1996, 1996

[38] R. Simmons. The curvature velocity method for local obstacle avoidance. In Proceedings of the International Conference on Robotics and Automation, pages 3375-3382, Minneapolis (USA), april 1996.

[39] P. Fiorini and Z. Shiller. Motion planning in dynamic environments using velocity obstacles. International Journal of Robotics Research, 17(7):760-772, July 1998.

[40] N.Y. Ko and R. Simmons. The lane-curvature method for local obstacle avoidance. In Proceedings of the IEEE/RSJ International Conference on Intelligent Robots and Systems, Victoria (Canada), October 1998.

[41] J. Minguez and L. Montano. Nearness diagram navigation (ND): A new real time collision avoidance approach for holonomic and no holonomic mobile robots. In Proceedings of the IEEE/RSJ International Conference on Intelligent Robots and Systems, Takamatsu, Japan, November.

[42] N. Roy and S. Thrun. Motion planning through policy search. In Proceedings of the IEEE/RSJ International Conference on Intelligent Robots and Systems, Lausanne, Switzerland, 2002.

[43] J. Minguez, L. Montano, and J. Santos-Victor. Reactive navigation for non-holonomic robots using the ego kinematic space. In Proceedings IEEE International Conference on Robotics and Automation, Washington (US), May 2002.

[44] Thierry Fraichard and Hajime Asama. Inevitable collision states - a step towards safer robots? Advanced Robotics, 18(10):1001-1024, 2004.

[45] S. LaValle and J. Kuffner. Randomized kinodynamic planning. In Proceedings of the IEEE International Conference on Robotics and Automation, volume 1, pages 473-479, Detroit (US), May 1999. 